\title{
Strong approximation of lacunary series with random gaps
}

\author{
Alina Bazarova ${ }^{1}$, Istvan Berkes ${ }^{2}$, and Marko Raseta ${ }^{3}$
}

\begin{abstract}
We investigate the asymptotic behavior of sums $\sum_{k=1}^{N} f\left(n_{k} x\right)$, where $f$ is a mean zero, smooth periodic function on $\mathbb{R}$ and $\left(n_{k}\right)_{k \geq 1}$ is a random sequence such that the gaps $n_{k+1}-n_{k}$ are i.i.d. Our result shows that, in contrast to the classical Salem-Zygmund theory, the almost sure behavior of lacunary series with random gaps can be described very precisely without any assumption on the size of the gaps.
\end{abstract}

\section{Introduction}

Let $f: \mathbb{R} \rightarrow \mathbb{R}$ be a measurable function satisfying

$$
f(x+1)=f(x), \quad \int_{0}^{1} f(x) d x=0, \quad\|f\|^{2}=\int_{0}^{1} f^{2}(x) d x<\infty .
$$

It is well known that for rapidly increasing $\left(n_{k}\right)_{k \geq 1}$ the sequence $\left(f\left(n_{k} x\right)\right)_{k \geq 1}$ behaves like a sequence of independent random variables over the probability space

2010 Mathematics Subject Classification. Primary 60F17, 42A55, $42 \mathrm{~A} 61$.

Keywords: lacunary series, random indices, Wiener approximation.

1) University of Warwick, Systems Biology Centre. Email: a.bazarova@warwick.ac.uk.

2) Alfréd Rényi Institute of Mathematics, Reáltanoda u. 13-15, 1053 Budapest, Hungary. Email: berkes.istvan@renyi.mta.hu. Research supported by FWF Grant P24302-N18 and NKFIH Grant K 108615.

3) University of Keele, Research Institute for Primary Care and Health Sciences and Research Institute for Applied Clinical Sciences. Email: m.raseta@keele.ac.uk. 
$([0,1], \mathcal{B}, \lambda)$, where $\mathcal{B}$ is the Borel $\sigma$-algebra and $\lambda$ is the Lebesgue measure. For example, if

$$
n_{k+1} / n_{k} \rightarrow \infty
$$

and $f$ satisfies the Lip $\alpha$ condition

$$
|f(x)-f(y)| \leq K|x-y|^{\alpha} \quad(x, y \in \mathbb{R})
$$

for some constants and $\alpha>0$ and $K>0$, then

$$
N^{-1 / 2} \sum_{k=1}^{N} f\left(n_{k} x\right) \stackrel{d}{\longrightarrow} N\left(0,\|f\|^{2}\right)
$$

and

$$
\limsup _{N \rightarrow \infty}(2 N \log \log N)^{-1 / 2} \sum_{k=1}^{N} f\left(n_{k} x\right)=\|f\| \quad \text { a.s. }
$$

with respect to $([0,1], \mathcal{B}, \lambda)$ (see Takahashi [24], [25]). Here, and in the sequel, $\|\cdot\|$ denotes the $L^{2}$ norm. Assuming only the Hadamard gap condition

$$
n_{k+1} / n_{k} \geq q>1, \quad k=1,2, \ldots
$$

the situation becomes more complicated. Kac [12] proved that $f\left(n_{k} x\right)$ satisfies the CLT for $n_{k}=2^{k}$ and Erdôs and Fortet (see [13], p. 646) showed that this generally fails for $n_{k}=2^{k}-1$. Gaposhkin [10] showed that $f\left(n_{k} x\right)$ satisfies the CLT provided the ratios $n_{k+1} / n_{k}$ are integers or $n_{k+1} / n_{k} \rightarrow \alpha>1$ where $\alpha^{r}$ is irrational for $r=1,2, \ldots$. A necessary and sufficient number-theoretic condition for the CLT for $f\left(n_{k} x\right)$ under (1.5) was given by Aistleitner and Berkes [4]. For a related sufficient criterion for the law of the iterated logarithm for the discrepancy of $\left\{n_{k} x\right\}$ for almost all $x$, see Aistleitner [1].

For subexponentially growing sequences $\left(n_{k}\right)$, the asymptotic behavior of $S_{N}=$ $\sum_{k=1}^{N} f\left(n_{k} x\right)$ becomes much more complicated and the arising number theoretical problems become essentially intractable. As a consequence, the limit distribution (if it exists) of normed sums of $f\left(n_{k} x\right)$ is not known even for $f(x)=\sin 2 \pi x$ and simple sequences like $n_{k}=k^{r}(r=3,4, \ldots)$. (In the case of $n_{k}=k^{2}$ the limit distribution was found using deep methods, see Jurkat and Van Horne [11], Marklof [14].) In such situations, it is natural to investigate the random case, i.e. when $\left(n_{k}\right)$ is an increasing random sequence, and prove asymptotic results valid for almost all $\left(n_{k}\right)$; in other words, to describe the "typical" behavior of sums $\sum_{k=1}^{N} f\left(n_{k} x\right)$. The simplest model for sequences with random gaps is when the gaps $n_{k+1}-n_{k}$ are i.i.d. random variables, and in a series of papers Schatte [19], [20], [21] gave a general study of this model. In particular, Schatte gave metric upper bounds for 
the discrepancy of $\left\{n_{k} x\right\}$ in a large class of discrete and continuous cases. Schatte's results have been extended and improved by Weber [26], Berkes and Weber [6], Berkes and Raseta [5]; on the other hand, Raseta [18] proved a functional law of the iterated logarithm for sums $\sum_{k=1}^{N} f\left(n_{k} x\right)$ for smooth periodic $f$. The purpose of the present paper is to prove that in the case of gaps $n_{k+1}-n_{k}$ with absolutely continuous distribution, the partial sums $\sum_{k=1}^{N} f\left(n_{k} x\right)$ can be closely approximated by a Wiener process, a result having far reaching asymptotic consequences for the sequence $f\left(n_{k} x\right)$. More precisely, we will prove the following result.

Theorem 1. Let $\left(X_{n}\right)_{n \geq 1}$ be a sequence of i.i.d. random variables defined on a probability space $(\Omega, \mathcal{F}, \mathbb{P})$ and let $S_{n}=\sum_{k=1}^{n} X_{k}$. Assume $X_{1}$ is bounded with bounded density. Let $f$ be a $\operatorname{Lip}(\alpha)$ function satisfying (1.1) and put

$$
A_{x, f}=\|f\|^{2}+2 \sum_{k=1}^{\infty} \mathbb{E} f(U) f\left(U+S_{k} x\right),
$$

where $U$ is a uniform $(0,1)$ random variable, independent of $\left(X_{n}\right)_{n \geq 1}$. Then for any fixed $x>0$ the series (1.6) is absolutely convergent with $\mathbb{P}$-probability $1, A_{x, f} \geq 0$ and the sequence $\left(X_{k}\right)_{k \geq 1}$ can be redefined, without changing its distribution, on a new probability space together with a Wiener process $W^{(x)}(\cdot)$ such that

$$
\sum_{k=1}^{n} f\left(S_{k} x\right)=W^{(x)}\left(A_{x, f} n\right)+O\left(n^{5 / 12+\varepsilon}\right) \quad \text { a.s. }
$$

for any $\varepsilon>0$.

Clearly, the redefinition of $\left(X_{k}\right)$ in Theorem 1 does not change the asymptotic properties of the sums $\sum_{k=1}^{N} f\left(S_{k} x\right)$ and thus limit theorems implied by the approximation (1.7) for the redefined sequence $f\left(S_{k} x\right)$ hold for the original sequence defined on $(\Omega, \mathcal{F}, \mathbb{P})$ as well.

We note that in Theorem 1 we do not assume $X_{1}>0$, and thus the sequence $\left(S_{k}\right)_{k \geq 1}$ need not be increasing. If $E X_{1}=0$, then by standard results of probability theory the sequence $\left(S_{k}\right)_{k \geq 1}$ is dense in $\mathbb{R}$; otherwise the random walk $\left(S_{k}\right)_{k \geq 1}$ is transient and $S_{k}$ tends to $+\infty$ or $-\infty$ almost linearly. The a.s. absolute convergence of the series in (1.6) will follow from the arguments in Section 4.

An immediate consequence of Theorem 1 is

$$
\limsup _{N \rightarrow \infty}(2 N \log \log N)^{-1 / 2} \sum_{k=1}^{N} f\left(S_{k} x\right)=A_{x, f}^{1 / 2}
$$

$\mathbb{P}$-a.s. for every fixed $x>0$. Thus by Fubini's theorem, with $\mathbb{P}$-probability 1 (i.e. for almost all sequences $\left(S_{k}\right)_{k \geq 1}$ generated by the random walk model), the sequence 
$f\left(S_{k} x\right)$ satisfies the LIL (1.8) for almost every $x \in \mathbb{R}$ with respect to the Lebesgue measure. Note that, in contrast to the LIL (1.4) in the nonrandom case, the limsup in (1.8) is a function of $x$. A similar argument yields an Chung type lower LIL for $f\left(S_{k} x\right)$, namely

$$
\liminf _{N \rightarrow \infty}\left(\frac{\log \log N}{N}\right)^{1 / 2} \max _{1 \leq M \leq N}\left|\sum_{k=1}^{M} f\left(S_{k} x\right)\right|=\frac{\pi}{\sqrt{8}} A_{x, f}^{1 / 2} \quad \text { a.s. }
$$

for almost all $x \in \mathbb{R}$ and for almost all sequences $\left(S_{k}\right)_{k \geq 1}$ generated by the random walk model. The functional versions of these results can also be written out and proved without any problem. For further asymptotic consequences of an approximation result of type (1.7) we refer to Strassen [22] and Philipp and Stout [17].

In view of (1.8) and (1.9), the properties of the function $A_{x, f}$ are of considerable interest and we will investigate them in Section 4.

Note that all of the previous consequences of Theorem 1 were almost sure limit theorems and using Fubini's theorem we cannot prove, e.g., that $\mathbb{P}$-a.s. the normed partial sums $\left(A_{x, f} n\right)^{-1 / 2} \sum_{k=1}^{n} f\left(S_{k} x\right)$ satisfy the central limit theorem over $([0,1], \mathcal{B}, \lambda)$. We now formulate a version of Theorem 1 implying a CLT and many related weak limit theorems.

Theorem 2. Under the conditions of Theorem 1 the sequence $\left(X_{k}\right)_{k \geq 1}$ can be redefined, without changing its distribution, on a new probability space together with a Wiener process $W$ such that

$$
\sum_{k=1}^{n} f\left(S_{k} \xi\right)=A_{\xi}^{1 / 2} W(n)+O\left(n^{5 / 12+\varepsilon}\right) \quad \text { a.s. }
$$

for any $\varepsilon>0$, where $\xi$ is a random variable uniformly distributed over $(0,1)$, independent of $\left(X_{k}\right)$ and $W$.

In other words, we can get an approximation of $\sum_{=1}^{N} f\left(n_{k} x\right)$ with a single Wiener process $W$ when not only the sequence $\left(n_{k}\right)_{k \geq 1}$, but also the $x$ is randomized. Theorem 2 implies, for example, that

$$
\frac{1}{\sqrt{n}} \sum_{k=1}^{n} f\left(S_{k} \xi\right) \stackrel{d}{\longrightarrow} N\left(0, A_{\xi}\right)
$$

where the right hand side denotes the distribution of $A_{\xi}^{1 / 2} \zeta$, where $\zeta$ is an $N(0,1)$ variable independent of $\xi$. Clearly, this distribution is mixed normal. However, this is a central limit theorem on the square $\Omega \times[0,1]$, and whether $n^{-1 / 2} \sum_{k=1}^{n} f\left(S_{k} x\right)$ has a mixed Gaussian limit $\mathbb{P}$-a.s. over $([0,1], \mathcal{B}, \lambda)$ remains open. 
As we see, the limsup resp. liminf in (1.8), (1.9) are functions of $x$, in contrast to constant limsup and liminf in the case of sums of independent random variables. Similarly, the limit distribution of normed partial sums in Theorem 2 is a mixed normal distribution, in contrast to standard Gaussian limit in classical situations. In the case of sums $\sum_{k=1}^{N} \sin n_{k} x$ with $n_{k}=2^{k}-1$, this phenomenon was discovered by Erdôs and Fortet (see [13], p. 646); for more general series see Morgenthaler [15], Weiss [27], Gaposhkin [10]. The deeper fact that the limsup in the law of the iterated logarithm for the discrepancy of lacunary sequences $\left\{n_{k} x\right\}$ can also be nonconstant, was proved by Aistleitner [2], [3] and Fukuyama [8], [9]. See also Berkes and Raseta [5] for the exact value of the limsup in case of the discrepancy of $\left\{n_{k} x\right\}$ for random $n_{k}$.

\section{Some lemmas}

In the Introduction we discussed implications of our theorems for the partial sums $\sum_{k=1}^{N} f\left(S_{k} x\right)$ as a sequence of random variables over different probability spaces. For the rest of the paper, $x>0$ will be fixed and we consider $f\left(S_{k} x\right)$ as a sequence of random variables over $(\Omega, \mathcal{F}, \mathbb{P})$, and the symbols $\mathbb{P}, \mathbb{E}$ will be meant with respect to this probability space.

Lemma 1 below, which is a slight generalization of Lemma 2 of [18], establishes the near independence of separated block sums of the variables $f\left(S_{k} x\right)$. The proof of the present form requires only routine changes.

Lemma 1. Assume the conditions of Theorem 1, let $\ell_{1}, \ell_{2}, \ldots$ be positive integers and let $I_{1}, I_{2}, \ldots$ be closed intervals with positive integer endpoints such that the left endpoint of $I_{k}$ exceeds the right endpoint of $I_{k-1}$ by at least $\ell_{k}$. Then there exists a sequence $\delta_{1}, \delta_{2}, \ldots$ of random variables satisfying the following properties:

(i) $\left|\delta_{k}\right| \leq C e^{-\lambda \ell_{k}}$ for all $k \in \mathbb{N}$, where $C$ and $\lambda$ are positive constants.

(ii) The random variables

$$
\sum_{i \in I_{1}} f\left(S_{i}\right), \sum_{i \in I_{2}} f\left(S_{i}-\delta_{1}\right), \ldots, \sum_{i \in I_{k}} f\left(S_{i}-\delta_{k-1}\right), \ldots
$$

are independent.

Put $\widetilde{m}_{k}=\sum_{j=1}^{k}\left\lfloor j^{1 / 2}\right\rfloor, \widehat{m}_{k}=\sum_{j=1}^{k}\left\lfloor j^{1 / 4}\right\rfloor$ and let $m_{k}=\widetilde{m}_{k}+\widehat{m}_{k}$. Using Lemma 1 we can construct sequences $\left(\Delta_{k}\right)_{k \geq 0},\left(\Pi_{k}\right)_{k \geq 0}$ of random variables such that $\Delta_{0}=0$, $\Pi_{0}=0$,

$$
\left|\Delta_{k}\right| \leq C e^{-\lambda k^{1 / 4}}, \quad\left|\Pi_{k}\right| \leq C e^{-\lambda \sqrt{k}} \quad(k \geq 1)
$$


and

$$
\begin{aligned}
& T_{k}:=\sum_{j=m_{k-1}+1}^{m_{k-1}+\lfloor\sqrt{k}\rfloor}\left(f\left(S_{j}-\Delta_{k-1}\right)-\mathbb{E} f\left(S_{j}-\Delta_{k-1}\right)\right) \quad(k \geq 1) \\
& T_{k}^{*}:=\sum_{j=m_{k-1}+\lfloor\sqrt{k}\rfloor+1}^{m_{k}}\left(f\left(S_{j}-\Pi_{k-1}\right)-\mathbb{E} f\left(S_{j}-\Pi_{k-1}\right)\right) \quad(k \geq 1)
\end{aligned}
$$

are sequences of independent, mean zero random variables.

Lemma 2. We have

$$
\sum_{k=1}^{n} \mathbb{E} T_{k}^{2}=A_{1} \widetilde{m}_{n}+O(n) \quad \sum_{k=1}^{n} \mathbb{E}\left(T_{k}^{*}\right)^{2}=A_{1} \widehat{m}_{n}+O(n),
$$

where $A_{1}=A_{1, f}$ is defined by (1.6) with $x=1$.

Lemma 2 implies $A_{1}=A_{1, f} \geq 0$ and similarly we have $A_{x, f} \geq 0$ for all $x>0$. The series expansion (1.6) resembles the series expansion of the long range variance of a stationary process. The weaker relations

$$
\sum_{k=1}^{n} \mathbb{E} T_{k}^{2} \sim A_{1} \widetilde{m}_{n} \quad \sum_{k=1}^{n} \mathbb{E}\left(T_{k}^{*}\right)^{2} \sim A_{1} \widehat{m}_{n}
$$

were proved in [18], Lemma 2. The proof of the present form uses the same argument with minor changes.

Lemma 3. We have

$$
\sup _{0 \leq t \leq 1}\left|\mathbb{P}\left(S_{k} \leq t\right)-t\right| \leq \rho^{k-2} \mathbb{E} X_{1}^{2} \quad(k \geq 2)
$$

where $S_{k}$ is meant mod 1 and $\rho=\sup _{r \in \mathbb{Z} \backslash\{0\}}\left|\mathbb{E}\left(e^{2 \pi i r X_{1}}\right)\right|$.

Since $X_{1}$ has a nonlattice distribution, $\nu_{r}=\left|\mathbb{E}\left(e^{2 \pi i r X_{1}}\right)\right|<1$ for any fixed integer $r \neq 0$ (see e.g. Feller [7], p. 501, Lemma 4). Also, $\lim _{r \rightarrow \infty} \nu_{r}=0$ by the RiemannLebesgue lemma and thus $\rho$ defined in Lemma 3 satisfies $\rho<1$.

Proof of Lemma 3. With $\mathbb{E} X_{1}^{2}$ replaced by an unspecified constant $C$ depending on the distribution of $X_{1}$, this lemma follows from statement (c) of Theorem 1 of Schatte [19]. To get $C=\mathbb{E} X_{1}^{2}$ we note that letting $p_{k}$ denote the density of $S_{k}$ and $f(r)=\mathbb{E}\left(e^{2 \pi i r X_{1}}\right)$, we have by a formula in the proof of Theorem 1 in Schatte [19], p. 277 and Parseval's relation

$$
\left|p_{n}(x)-1\right| \leq|f(r)|^{n-2} \sum_{r}|f(r)|^{2} \leq \rho^{n-2} \mathbb{E} X_{1}^{2} .
$$


Relation (2.3) implies that there exists a random variable $U^{*}$ on $(\Omega, \mathcal{F}, \mathcal{P})$, uniformly distributed over $(0,1)$, such that $\left|S_{k}-U^{*}\right| \leq \rho^{n-2} \mathbb{E} X_{1}^{2}$ and thus by the Lipschitz property of $f$ we have $\left|f\left(S_{k}\right)-f\left(U^{*}\right)\right|=O\left(\rho^{k}\right)$. Since $\mathbb{E} f\left(U^{*}\right)=\int_{0}^{1} f(x) d x=0$ by (1.1), we have proved

$$
\mathbb{E} f\left(S_{k}\right)=O\left(\rho^{k}\right) .
$$

The following lemma is a special case of Strassen's strong approximation theorem [23], Theorem 4.4 .

Lemma 4. Let $Y_{1}, Y_{2}, \ldots$ be independent r.v.'s with mean 0 and finite fourth moments, let $a_{n}=\sum_{i=1}^{n} \mathbb{E} Y_{i}^{2}$ and assume

$$
\sum_{n=1}^{\infty} \mathbb{E} Y_{n}^{4} / a_{n}^{2 \vartheta}<\infty
$$

with $0<\vartheta<1$. Then the sequence $Y_{1}, Y_{2}, \ldots$ can be redefined on a new probability space together with a Wiener process $W$ such that

$$
Y_{1}+\cdots+Y_{n}=W\left(a_{n}\right)+o\left(a_{n}^{(1+\vartheta) / 4} \log a_{n}\right) \quad \text { a.s. }
$$

\section{Proof of the theorems}

We begin with the proof of Theorem 1 . In what follows, $C_{1}, C_{2}, \ldots$ denote positive constants, depending (at most) on the distribution of $X_{1}$. Since together with $\left(X_{k}\right)$ the sequence $\left(X_{k} x\right)$ also satisfies the conditions of Theorem 1 for any $x>0$, it suffices to prove the theorem for $x=1$. We will apply Lemma 4 for the sequences $\left(T_{k}\right)_{k \geq 1}$ and $\left(T_{k}{ }^{*}\right)_{k \geq 1}$ defined before. Clearly, $\left(T_{k}\right)_{k \geq 1}$ is a sequence of independent, mean zero random variables and $\left|T_{k}\right| \leq M \sqrt{k}$, where $M=\sup _{x \in \mathbb{R}}|f(x)|$. In [6], pp. 59-60 it is shown that for arbitrary real coefficients $\left(c_{k}\right)$ we have

$$
\mathbb{E}\left(\sum_{k=1}^{N} c_{k} \xi_{k}\right)^{4} \leq C_{1}\left(\sum_{k=1}^{N} c_{k}^{2}\right)^{2}
$$

where $\xi_{k}=f\left(S_{k}\right)-\mathbb{E} f\left(S_{k}\right)$. By the Lipschitz property of $f$ and (2.1), replacing $S_{j}-\Delta_{k-1}$ by $S_{j}$ in the definition of $T_{k}$ results in an error of $O\left(\sqrt{k} \exp \left(-C_{2} k^{1 / 4}\right)\right)$ and thus using (3.1) we get

$$
\mathbb{E} T_{k}^{4} \leq C_{3} k
$$

Thus by $m_{k} \sim \widetilde{m}_{k} \sim \frac{2}{3} k^{3 / 2}$ we have

$$
\sum_{k \in \mathbb{N}} \frac{\mathbb{E} T_{k}^{4}}{\widetilde{m}_{k}^{4 / 3+2 \varepsilon}}<\infty
$$


for all $\varepsilon>0$ and thus using Lemma 2 and Lemma 4 with $\vartheta=2 / 3+\varepsilon,\left|\widetilde{m}_{n}-m_{n}\right|=$ $O\left(n^{5 / 4}\right)$ and standard fluctuation properties of the Wiener process it follows that after redefining the sequence $\left(X_{k}\right)_{k \geq 1}$ on a suitable new probability space we have

$$
\begin{aligned}
\sum_{k=1}^{n} T_{k} & =W\left(A_{1} \widetilde{m}_{n}+O(n)\right)+O\left(\widetilde{m}_{n}^{\left(1+\frac{2}{3}+\varepsilon\right) / 4} \log \widetilde{m}_{n}\right) \\
& =W\left(A_{1} m_{n}\right)+O\left(n^{5 / 8} \log n\right)+O\left(m_{n}^{\left(1+\frac{2}{3}+\varepsilon\right) / 4} \log m_{n}\right) \\
& =W\left(A_{1} m_{n}\right)+O\left(m_{n}^{\left(1+\frac{2}{3}+\varepsilon\right) / 4} \log m_{n}\right) \quad \text { a.s. }
\end{aligned}
$$

for some Wiener process $W$. Define a sequence $(p(n))_{n \geq 1}$ of integers by

$$
m_{p(n)} \leq n<m_{p(n)+1}
$$

Clearly, $p(n) \sim C_{4} n^{2 / 3}$ and, as we have shown above,

$$
\sum_{k=1}^{p(n)} T_{k}=W\left(A_{1} m_{p(n)}\right)+O\left(m_{p(n)}\left(1+\frac{2}{3}+\varepsilon\right) / 4 \log m_{p(n)}\right) \quad \text { a.s. }
$$

and similarly

$$
\sum_{k=1}^{p(n)} T_{k}{ }^{*}=W^{\prime}\left(A_{1} \widehat{m}_{p(n)}\right)+O\left(\widehat{m}_{p(n)}^{\left(1+\frac{3}{5}+\delta\right) / 4} \log \widehat{m}_{p(n)}\right) \quad \text { a.s. }
$$

for some other Brownian motion $W^{\prime}$. Now

$$
\begin{aligned}
\sum_{k=1}^{n} f\left(S_{k}\right)= & \sum_{k=1}^{p(n)} T_{k}+\sum_{k=1}^{p(n)} T_{k}^{*}+\sum_{k=1}^{p(n)} \sum_{j=m_{k-1}+1}^{m_{k-1}+\lfloor\sqrt{k}\rfloor}\left(f\left(S_{j}\right)-f\left(S_{j}-\Delta_{k-1}\right)+\mathbb{E} f\left(S_{j}-\Delta_{k-1}\right)\right) \\
& +\sum_{k=1}^{p(n)} \sum_{j=m_{k-1}+\lfloor\sqrt{k}\rfloor+1}^{m_{k}}\left(f\left(S_{j}\right)-f\left(S_{j}-\Pi_{k-1}\right)+\mathbb{E} f\left(S_{j}-\Pi_{k-1}\right)\right) \\
& +\sum_{k=m_{p(n)}+1}^{n} f\left(S_{k}\right) .
\end{aligned}
$$

Thus

$$
\begin{aligned}
\left|\sum_{k=1}^{n} f\left(S_{k}\right)-W\left(A_{1} n\right)\right| & \leq\left|\sum_{k=1}^{p(n)} T_{k}-W\left(A_{1} m_{p(n)}\right)\right|+\left|W\left(A_{1} m_{p(n)}\right)-W\left(A_{1} n\right)\right| \\
& +\left|\sum_{k=1}^{p(n)} T_{k}^{*}-W^{\prime}\left(A_{1} \widehat{m}_{p(n)}\right)\right|+\left|W^{\prime}\left(A_{1} \widehat{m}_{p(n)}\right)\right|
\end{aligned}
$$




$$
\begin{aligned}
& +\left|\sum_{k=1}^{p(n)} \sum_{j=m_{k-1}+1}^{m_{k-1}+\lfloor\sqrt{k}\rfloor}\left(f\left(S_{j}\right)-f\left(S_{j}-\Delta_{k-1}\right)+\mathbb{E} f\left(S_{j}-\Delta_{k-1}\right)\right)\right| \\
& +\left|\sum_{k=1}^{p(n)} \sum_{j=m_{k-1}+\lfloor\sqrt{k}\rfloor+1}^{m_{k}}\left(f\left(S_{j}\right)-f\left(S_{j}-\Pi_{k-1}\right)+\mathbb{E} f\left(S_{j}-\Pi_{k-1}\right)\right)\right| \\
& +\left|\sum_{k=m_{p(n)+1}}^{n} f\left(S_{k}\right)\right| .
\end{aligned}
$$

We estimate each term separately. Since $m_{p(n)} \sim n$, we have by $(3.2)$

$$
\left|\sum_{k=1}^{p(n)} T_{k}-W\left(A_{1} m_{p(n)}\right)\right|=o\left(m_{p(n)}^{(1+2 / 3+\varepsilon) / 4} \log m_{p(n)}\right)=o\left(n^{5 / 12+\varepsilon}\right) .
$$

Further $W\left(A_{1} m_{p(n)}\right)-W\left(A_{1} n\right)$ has distribution $N\left(0, A_{1}\left(n-m_{p(n)}\right)\right)$ and here

$$
n-m_{p(n)} \leq\left\lfloor(p(n)+1)^{1 / 2}\right\rfloor+\left\lfloor(p(n)+1)^{1 / 4}\right\rfloor=O\left(n^{1 / 3}\right) .
$$

Thus

$$
\mathbb{P}\left(\left|W\left(A_{1} m_{p(n)}\right)-W\left(A_{1} n\right)\right| \geq n^{7 / 24}\right) \leq 1-\Phi\left(C_{5} n^{1 / 4}\right)=O\left(n^{-2}\right)
$$

and hence the Borel-Cantelli lemma implies

$$
\left|W\left(A_{1} m_{p(n)}\right)-W\left(A_{1} n\right)\right|=O\left(n^{7 / 24}\right) \quad \text { a.s. }
$$

Also $\widehat{m}_{n} \sim C_{6} n^{5 / 4}$, hence $\widehat{m}_{p(n)} \sim C_{7} n^{5 / 6}$ and thus (3.3) yields

$$
\left|\sum_{k=1}^{p(n)} T_{k}^{*}-W^{\prime}\left(A_{1} \widehat{m}_{p(n)}\right)\right|=O\left(n^{5 / 12}\right) \quad \text { a.s. }
$$

Using the distribution of $W^{\prime}\left(A_{1} \widehat{m}_{p(n)}\right)$ and $\widehat{m}_{p(n)} \sim C_{7} n^{5 / 6}$, the Borel-Cantelli lemma yields

$$
\left|W^{\prime}\left(A_{1} \widehat{m}_{p(n)}\right)\right|=O\left(n^{5 / 12+\varepsilon}\right) \quad \text { a.s. }
$$

In view of $(2.1),(2.4)$ and the Lipschitz property of $f$ we have

$$
\begin{aligned}
& \left|\sum_{k=1}^{p(n)} \sum_{j=m_{k-1}+1}^{m_{k-1}+\lfloor\sqrt{k}\rfloor}\left(f\left(S_{j}\right)-f\left(S_{j}-\Delta_{k-1}\right)+\mathbb{E} f\left(S_{j}-\Delta_{k-1}\right)\right)\right| \\
& \leq \sum_{k=1}^{p(n)} \sum_{j=m_{k-1}+1}^{m_{k-1}+\lfloor\sqrt{k}\rfloor} C_{8} e^{-C_{9}(k-1)^{1 / 4}} \leq C_{8} \sum_{k=1}^{p(n)} \sqrt{k} e^{-C_{9}(k-1)^{1 / 4}}=O(1) .
\end{aligned}
$$


Similarly

$$
\mid \sum_{k=1}^{p(n)} \sum_{j=m_{k-1}+\lfloor\sqrt{k}\rfloor+1}^{m_{k}}\left(f\left(S_{j}\right)-f\left(S_{j}-\Pi_{k-1}\right)+\mathbb{E} f\left(S_{j}-\Pi_{k-1}\right) \mid=O(1) \quad\right. \text { a.s. }
$$

Finally, by (3.4)

$$
\left|\sum_{k=m_{p(n)}+1}^{n} f\left(S_{k}\right)\right| \leq C_{10}\left(n-m_{p(n)}\right)=O\left(n^{1 / 3}\right) .
$$

Summarizing the above estimates, we obtain our result.

Proof of Theorem 2. By the theorem of Ionescu Tulcea (see e.g. [16], p. 154), on a suitable probability space one can define jointly a sequence $\left\{X_{k}^{*}, k \geq 1\right\}$ of r.v.'s, a Wiener process $W^{*}$ and a r.v. $\xi$ uniformly distributed over $(0,1)$ such that the conditional distribution of the vector $\left(\left\{X_{k}^{*}, k \geq 1\right\}, W^{*}\right) \in \mathbb{R}^{\infty} \times C(0, \infty)$ given $\xi=x$ equals the distribution of the vector $\left(\left\{X_{k}, k \geq 1\right\}, W^{(x)}\right)$ in Theorem 1 . In particular, the conditional distribution of $\left\{X_{k}^{*}, k \geq 1\right\}$ given $\xi=x$ equals the distribution of $\left\{X_{k}^{*}, k \geq 1\right\}$ which does not depend on $x$ and thus $\xi$ is independent of $\left\{X_{k}^{*}, k \geq 1\right\}$. For the same reason, $\xi$ is independent of $W^{*}$. Further, by the construction and relation (1.7) of Theorem 1, we have the analogue of (1.10) where $S_{k}$ is replaced by the partial sums $S_{k}^{*}=\sum_{j=1}^{k} X_{j}^{*}$ and $W(n)$ is replaced by $W^{*}(n)$. This completes the proof of Theorem 2 .

\section{Properties of $A_{x, f}$}

In view of (1.8), (1.9), the function $A_{x, f}$ in (1.6) plays an important role in the asymptotic study of $\sum_{k=1}^{N} f\left(n_{k} x\right)$. In this section we study the properties of $A_{x, f}$. First we give an explicit formula for $A_{x, f}$ in the case $f(x)=\sin 2 \pi x$. Let $X_{1}^{*}=$ $X_{1}-\mu$, where $\mu=\mathbb{E} X_{1}$. Since $\mathbb{E} X_{1}^{*}=0$ and since all moments of $X_{1}^{*}$ exist by the boundedness of $X_{1}$, the Taylor expansion of the characteristic function $\varphi$ of $X_{1}^{*}$ around 0 is

$$
\varphi(t)=1+\sum_{k=2}^{\infty} \frac{(i t)^{k}}{k !} \mathbb{E}\left(X_{1}^{*}\right)^{k}
$$

where the even order terms give the real part and the odd order terms give the imaginary part. Grouping the even and odd terms, we get

$$
\varphi(2 \pi x)=1+B(x) x^{2}+i C(x) x^{3},
$$

where $B(x)=b_{0}+b_{2} x^{2}+\ldots$ and $C(x)=c_{0}+c_{2} x^{2}+\ldots$, here $b_{0}=-2 \pi^{2} \sigma^{2}$, where $\sigma^{2}$ is the variance of $X_{1}$ and $c_{0}=-\frac{4 \pi^{2}}{3} \mathbb{E}\left(X_{1}-\mu\right)^{3}$. 
Lemma 5. We have

$$
A_{x, f}=-\frac{1}{2}+\frac{K}{L}
$$

where

$$
K=1-\left(1+B(x) x^{2}\right) \cos 2 \pi \mu x+C(x) x^{3} \sin 2 \pi \mu x
$$

and

$$
\begin{aligned}
L=\left[1-\left(1+B(x) x^{2}\right)\right. & \left.\cos 2 \pi \mu x+C(x) x^{3} \sin 2 \pi \mu x\right]^{2} \\
+ & {\left[\left(1+B(x) x^{2}\right) \sin 2 \pi \mu x+C(x) x^{3} \cos 2 \pi \mu x\right]^{2} . }
\end{aligned}
$$

As a consequence, $A_{x, f}$ is infinitely many times differentiable for $x>0$ and

$$
\lim _{x \rightarrow 0} A_{x, f}=\left\{\begin{array}{cc}
\frac{\sigma^{2}}{2 \mu^{2}} & \text { if } \mu \neq 0, \\
+\infty & \text { if } \mu=0 .
\end{array}\right.
$$

Proof. Let $Z_{k}=S_{k} / \sqrt{k}, Z_{k}^{*}=\left(S_{k}-\mathbb{E} S_{k}\right) / \sqrt{k}=Z_{k}-\mu \sqrt{k}$. Then for $f(x)=\sin 2 \pi x$ we get, using the independence of $U$ and $S_{k}$,

$$
\begin{aligned}
& \mathbb{E} f(U) f\left(U+S_{k} x\right) \\
& =\mathbb{E} \sin (2 \pi U) \sin \left(2 \pi\left(U+S_{k} x\right)\right) \\
& =\mathbb{E} \sin ^{2}(2 \pi U) \cos \left(2 \pi S_{k} x\right)+\mathbb{E} \sin (2 \pi U) \cos (2 \pi U) \sin \left(2 \pi S_{k} x\right) \\
& =\mathbb{E} \sin ^{2}(2 \pi U) \mathbb{E} \cos \left(2 \pi S_{k} x\right)+\mathbb{E} \sin (2 \pi U) \cos (2 \pi U) \mathbb{E} \sin \left(2 \pi S_{k} x\right) \\
& =\frac{1}{2} \mathbb{E} \cos \left(2 \pi S_{k} x\right)=\frac{1}{2} \operatorname{Re}\left\{\mathbb{E} e^{2 \pi i S_{k} x}\right\}=\frac{1}{2} \operatorname{Re}\left\{\mathbb{E} e^{2 \pi i\left(S_{k}-\mu k\right) x} \cdot e^{2 \pi i \mu k x}\right\} \\
& =\frac{1}{2} \operatorname{Re}\left\{\varphi^{k}(2 \pi x) e^{2 \pi i \mu k x}\right\} .
\end{aligned}
$$

In the $\operatorname{Re}\{\ldots\}$ in the last line we have a geometric progression with quotient $q=$ $\varphi(2 \pi x) e^{2 \pi i \mu x}$. Since $X_{1}^{*}$ has a density, $|q|=|\varphi(2 \pi x)|<1$ for all $x>0$ and thus $\sum_{k=0}^{\infty} q^{k}$ is finite and we get

$$
\sum_{k=0}^{\infty} \mathbb{E} f(U) f\left(U+S_{k} x\right)=\frac{1}{2} \operatorname{Re}\left\{\frac{1}{1-\varphi(2 \pi x) e^{2 \pi i \mu x}}\right\} .
$$

Since the term for $k=0$ of the sum in (4.3) is equal to $\mathbb{E} f^{2}(U)=1 / 2$, we have

$$
\begin{aligned}
& A_{x, f}=1 / 2+2 \sum_{k=1}^{\infty} \mathbb{E} f(U) f\left(U+S_{k} x\right)=-1 / 2+2 \sum_{k=0}^{\infty} \mathbb{E} f(U) f\left(U+S_{k} x\right) \\
& =-\frac{1}{2}+\operatorname{Re}\left\{\frac{1}{1-\varphi(2 \pi x) e^{2 \pi i \mu x}}\right\} .
\end{aligned}
$$


Substituting (4.1) and $e^{2 \pi i \mu x}=\cos 2 \pi \mu x+i \sin 2 \pi \mu x$ into (4.4) we get, after some algebra, that the $\operatorname{Re}\{\ldots\}$ in the second line of (4.4) equals $K / L$, where $K$ and $L$ are defined above. Clearly, for $\mu \neq 0$ the Taylor series of $K$ and $L$ start with the term $\left(2 \pi^{2} \mu^{2}-b_{0}\right) x^{2}$, resp. $4 \pi^{2} \mu^{2} x^{2}$, and thus the limit of $\operatorname{Re}\{\cdots\}$ in the second line of (4.4) as $x \rightarrow 0$ is

$$
\frac{2 \pi^{2} \mu^{2}-b_{0}}{4 \pi^{2} \mu^{2}}=\frac{1}{2}+\frac{\sigma^{2}}{2 \mu^{2}}
$$

Thus, in view of (4.4) we get the first line of (4.2). For $\mu=0$ the expansion of $L$ starts with a term later than $x^{2}$ and we get the second line of (4.2). Since $X_{1}$ has a density, $|\varphi(2 \pi x)|<1$ for $x>0$ and the boundedness of $X_{1}$ implies that all moments of $X_{1}$ are finite. Thus the characteristic function $\varphi$ is infinitely many times differentiable, and consequently the right hand side of (4.3) and thus also $A_{x, f}$ are infinitely many times differentiable on $(0,+\infty)$.

Finally, we study the properties of $A_{x, f}$ for general smooth $f$.

Lemma 6. Assume that $X_{1}$ has a bounded density with a bounded derivative. Then the function $A_{x, f}$ is a continuous function of $x$ for all $x>0$ and $\lim _{x \rightarrow \infty} A_{x, f}=\|f\|^{2}$.

Proof. Applying Lemma 3 for the random variable $\widehat{X}_{1}=X_{1} x$ it follows that

$$
\sup _{0 \leq t \leq 1}\left|\mathbb{P}\left(S_{k} x \leq t\right)-t\right| \leq \mu_{2} x^{2} \rho_{x}^{k-2} \quad \text { where }
$$

where again, $S_{k} x$ is meant $\bmod 1$ and

$$
\rho_{x}=\sup _{r \in \mathbb{Z} \backslash\{0\}}|\psi(r x)|, \quad \psi(s)=\mathbb{E}\left(e^{2 \pi i s X_{1}}\right), \quad \mu_{2}=E X_{1}^{2} .
$$

Using the assumptions on the density $p$ of $X_{1}$ and integration by parts, we see that

$$
|\psi(s)|=\left|\int_{0}^{1} e^{2 \pi i s x} p(x) d x\right| \leq C_{11} s^{-1} \quad(s>0),
$$

and thus the right hand side of (4.5) cannot exceed

$$
\mu_{2} x^{2}\left(C_{11} / x\right)^{k-2}=\mu_{2} C_{11}^{2}\left(C_{11} / x\right)^{k-4} \quad(k \geq 4) .
$$

Fix now $0<A<C_{11}$. Clearly $\psi(s)$ is continuous for all $s$ and as we noted earlier, $|\psi(s)|<1$ for any $s \neq 0$ and $\lim _{s \rightarrow \infty}|\psi(s)|=0$. Thus for $x>A$ we have $|\psi(x)| \leq$ $1-\delta_{A}$, where $\delta_{A}$ is a positive number depending on $A$. Now if $x \geq 2 C_{11}$, the expression in (4.8) decreases exponentially in $k$, uniformly in $x$. On the other hand, if $A<x<2 C_{11}$, then $\rho_{x} \leq 1-\delta_{A}$ and thus the right hand side of (4.5) is bounded 
by $\mu_{2}\left(2 C_{11}\right)^{2}\left(1-\delta_{A}\right)^{k-2}$ and again we have exponential decay in $k$, uniformly in $x$. Thus (4.5), together with the Lipschitz property of $f$, implies that

$$
\left|\mathbb{E} f\left(u+S_{k} x\right)-\mathbb{E} f(u+V)\right| \leq C_{12}\left(\rho^{\prime}\right)^{k}
$$

for any $0 \leq u \leq 1$ and $x \geq A$ where $C_{12}$ and $\rho^{\prime}<1$ are positive constants also depending on $A$ and the distribution of $X_{1}$. Multiplying the last inequality with $f(u)$ and integrating with respect to $u$ we get

$$
\left|\mathbb{E} f(U) f\left(U+S_{k} x\right)-\mathbb{E} f(U) f(U+V)\right| \leq C_{13}\left(\rho^{\prime}\right)^{k}
$$

for $x \geq A$ where $\mathrm{U}$ is a uniform $(0,1)$ random variable independent of $V$ and $S_{k}$. Since

$$
\mathbb{E} f(U) f(U+V)=\int_{0}^{1} \int_{0}^{1} f(u) f(u+v) d u d v=0
$$

by (1.1), we proved that uniformly for $x \geq A$, the terms of the sum in (1.6) decrease exponentially and thus the sum converges uniformly over $[A, \infty)$. But by the Lipschitz property of $f$ all terms of the sum in (1.6) are continuous functions of $x$, the sum of the series and thus $A_{x, f}$ also are continuous over $[A, \infty)$ and since $A>0$ was arbitrary, $A_{x, f}$ is continuous over $(0, \infty)$, as stated.

Next we prove that for any fixed $k \geq 1$ we have

$$
\lim _{x \rightarrow \infty} \mathbb{E} f(U) f\left(U+S_{k} x\right)=0,
$$

i.e. every term of the sum in (1.6) tends to 0 as $x \rightarrow \infty$. Since the series converges uniformly over $[A, \infty)$ for any $A>0$, this implies that its sum also converges to 0 as $x \rightarrow \infty$, i.e. $\lim _{x \rightarrow \infty} A_{x, f}=\|f\|^{2}$. As before, (4.9) will follow if we show that $\mathbb{E} f\left(u+S_{k} x\right) \rightarrow 0$ as $x \rightarrow \infty$ for any fixed $k \geq 1$ and any $u \in(0,1)$. Since, together with the function $f(x)$, the function $f(x+u)$ also satisfies (1.1), it remains to show that for any fixed $k \geq 1$ we have $\mathbb{E} f\left(S_{k} x\right) \rightarrow 0$, or equivalently

$$
\int_{0}^{1} f(u x) g_{k}(u) d u \longrightarrow 0 \quad \text { as } x \rightarrow \infty
$$

where $g_{k}$ is the density of $S_{k}$. To this end we first note that if $h(u)$ is the indicator function of a subinterval $(a, b)$ of $(0,1)$, then

$$
\int_{0}^{1} f(u x) h(u) d u \longrightarrow 0 \quad \text { as } x \rightarrow \infty
$$

This is clear, since $\int_{a}^{b} f(u x) d u=x^{-1} \int_{a x}^{b x} f(v) d v$ and because of (1.1) the last integral is bounded by $2 \int_{0}^{1}|f(v)| d v$. It follows then that (4.11) holds for any stepfunction in $(0,1)$ and thus by a simple approximation argument, for any bounded measurable function in $(0,1)$. This proves (4.10) and thus (4.9) is established, completing the proof of Lemma 6. 


\section{References}

[1] C. Aistleitner. On the law of the iterated logarithm for the discrepancy of lacunary sequences. Trans. Amer. Math. Soc. 362 (2010), 5967-5982.

[2] C. Aistleitner. Irregular discrepancy behavior of lacunary series. Monatsh. Math. 160 (2010), 1-29.

[3] C. Aistleitner. Irregular discrepancy behavior of lacunary series II. Monatsh. Math. 161 (2010), 255-270.

[4] C. Aistleitner and I. Berkes, On the central limit theorem for $f\left(n_{k} x\right)$. Prob. Theory Rel. Fields 146 (2010), 267-289.

[5] I. Berkes and M. RAseta On the discrepancy and empirical distribution function of $\left\{n_{k} \alpha\right\}$. Unif. Distr. Theory 10 (2015), 1-17.

[6] I. Berkes and M. Weber. On the convergence of $\sum c_{k} f\left(n_{k} x\right)$. Mem. Amer. Math. Soc. 201 (2009), no. 943, viii+72 pp.

[7] W. FELLER. An introduction to probability theory and its applications, Vol II. Wiley, 1970.

[8] K. Fukuyama. A law of the iterated logarithm for discrepancies: non-constant limsup. Monatsh. Math. 160 (2010), 143-149.

[9] K. Fukuyama and S. Miyamoto. Metric discrepancy results for Erdős-Fortet sequence. Studia Sci. Math. Hungar. 49 (2012), 52-78.

[10] V. F. Gaposhkin. Lacunary series and independent functions. Russian Math. Surveys 21/6 (1966), 1-82.

[11] W. B. Jurkat, J. W. Van Horne, The proof of the central limit theorem for theta sums. Duke Math. J. 48 (1981), 873-885.

[12] M. KAC, On the distribution of values of sums of the type $\sum f\left(2^{k} t\right)$. Ann. of Math. 47 (1946), 33-49.

[13] M. KAC, Probability methods in some problems of analysis and number theory, Bull. Amer. Math. Soc. 55 (1949), 641-665.

[14] J. Marklof, Limit theorems for theta sums. Duke Math. J. 97 (1999), 127153. 
[15] W. Morgenthaler. A central limit theorem for uniformly bounded orthonormal systems. Trans. Amer. Math. Soc. 79 (1955), 281-311.

[16] P. Neveu, Bases mathématiques du calcul des probabilités, second ed., Masson et Cie, Paris, 1970.

[17] W. Phlipp and W.F. Stout, Almost sure invariance principles for partial sums of weakly dependent random variables. Mem. Amer. Math. Soc. 161 (1975), iv +140 pp.

[18] M. Raseta, On lacunary series with random gaps. Acta Math. Hungar. 144 (2014), 150-161.

[19] P. Schatte, On the asymptotic uniform distribution of sums reduced mod 1, Math. Nachr. 115 (1984), 275-281.

[20] P. Schatte, On a law of the iterated logarithm for sums mod 1 with applications to Benford's law, Prob. Theory Rel. Fields 77 (1988), 167-178.

[21] P. Schatte, On a uniform law of the iterated logarithm for sums mod 1 and Benford's law. Lithuanian Math. J. 31 (1991), 133-142.

[22] V. Strassen. An invariance principle for the law of the iterated logarithm. Zeitschrift für Wahrsch. verw. Geb. 3 (1964), 211-226.

[23] V. Strassen Almost sure behavior of sums of independent random variables and martingales. Proc. Fifth Berkeley Sympos. Math. Statist. and Probability, Vol. II, Part 1, pp. 315-343. Univ. California Press, 1967.

[24] S. TAkAhashi A gap sequence with gaps bigger than the Hadamards. Tohoku Math. J. 13 (1961). 105-111.

[25] S. TAkAhashi, The law of the iterated logarithm for a gap sequence with infinite gaps. Tohoku Math. J. 15 (1963), 281-288.

[26] M. Weber, Discrepancy of randomly sampled sequences of integers, Math. Nachr. 271 (2004), 105-110.

[27] M. Weiss, On the law of the iterated logarithm for uniformly bounded orthonormal systems. Trans. Amer. Math. Soc. 92 (1959) 531-553. 